\title{
D iagnóstico etiológico da trombose venosa profunda de repetição dos membros inferiores
}

Etiologic diagnosis of recurrent deep venous thrombosis of the lower limbs

\author{
Eduardo Lichtenfels ${ }^{1}$, Aline S. Becker ${ }^{1}$, Vinicius C. Pires ${ }^{1}$, Telmo Pedro Bonamigo
}

\section{Parte II - 0 que foi feito}

As provas de coagulação, exames laboratoriais rotineiros e radiografia de tórax eram normais.

O s exames laboratoriais para avaliação de trombofilia (proteínaC eS, fator V deLeiden, anticardiolipina e fator lúpico) eram normais.

A paciente foi submetida, então, a uma tomografia computadorizada de abdômen e, posteriormente, a uma venografia de cava inferior.

A tomografia computadorizada de abdômen (Figura 1) não evidenciou nenhuma massa abdominal ou lesões orgânicas. 0 laudo descreve: "O bserva-se que o segmento infra-renal da veia cava inferior está em topografia habitual eausência do segmento supra-renal da veia cava inferior, incluindo o segmento intrahepático. Após a veia cava inferior receber as renais, a mesma tem continuidade através da veia ázigo, que apresenta calibre bastante aumentado. As veias hepáticas desembocam diretamente no átrio direito".

A venografia dacavainferior (Figuras 2 e 3 ) evidenciou a agenesia do segmento supra-renal da veia cava inferior. O laudo cita: "O segmento infra-renal da veia cava continua-se cranialmente pela veia ázigo, que se

1. Residente de Cirurgia Vascular, Serviço de Angiologia e Cirurgia Vas cular, Irmandade Santa Casa de Misericórdia de Porto Alegre, FFFCMPA Porto Alegre, RS.

2. Professor titular, Disciplina de Cirurgia Vascular, Fundação Faculdade Federal de Ciências Médicas de Porto Alegre (FFFCMPA), Porto Alegre, RS. Chefe, Serviço de Cirurgia Vascular, Irmandade Santa Casa de Misericórdia de Porto Alegre, Porto Alegre, RS.

\section{Artigo submetido em 05.03.06, aceito em 13.06.06.}

encontra vicariante. Visualiza-se circulação venosa intra-renal através da veia do lobo caudado e veias suprahepáticas quedrenam diretamentepara o átrio direito".
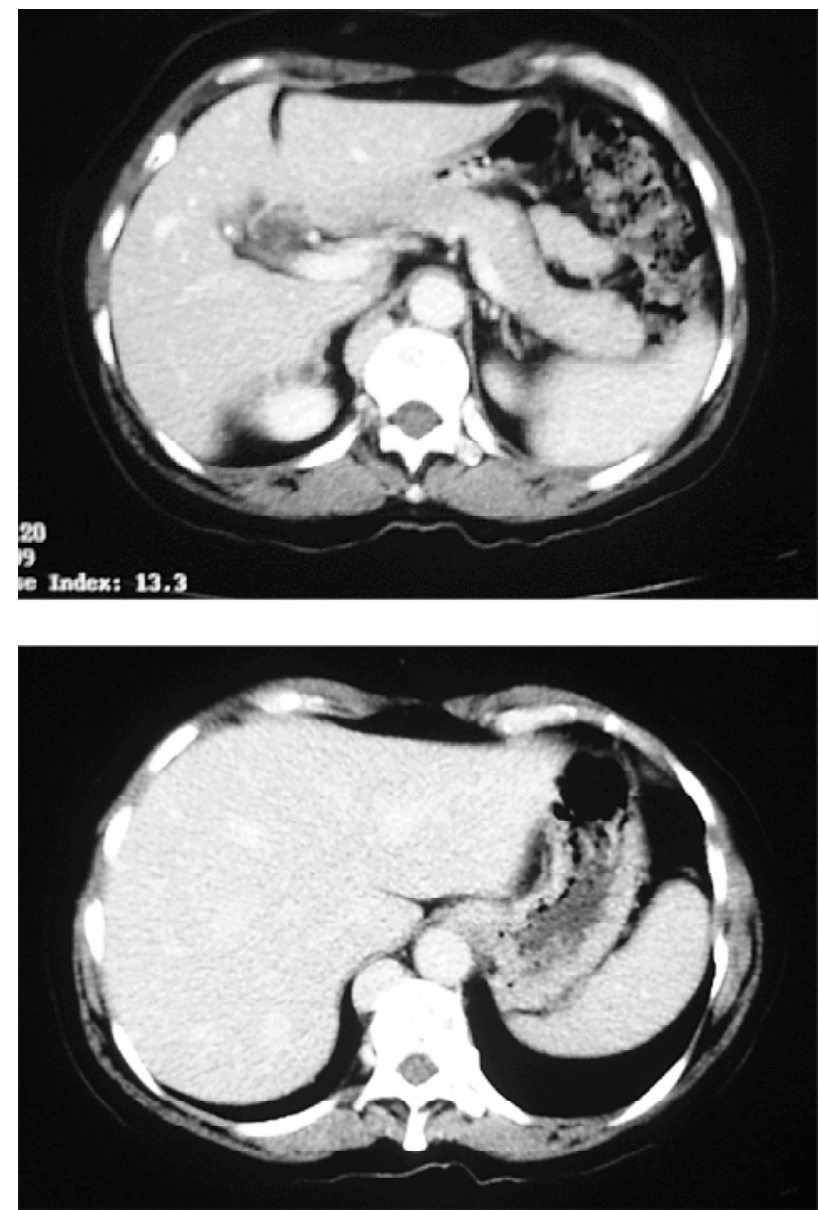

Figura 1 - Tomografia computadorizada abdominal 


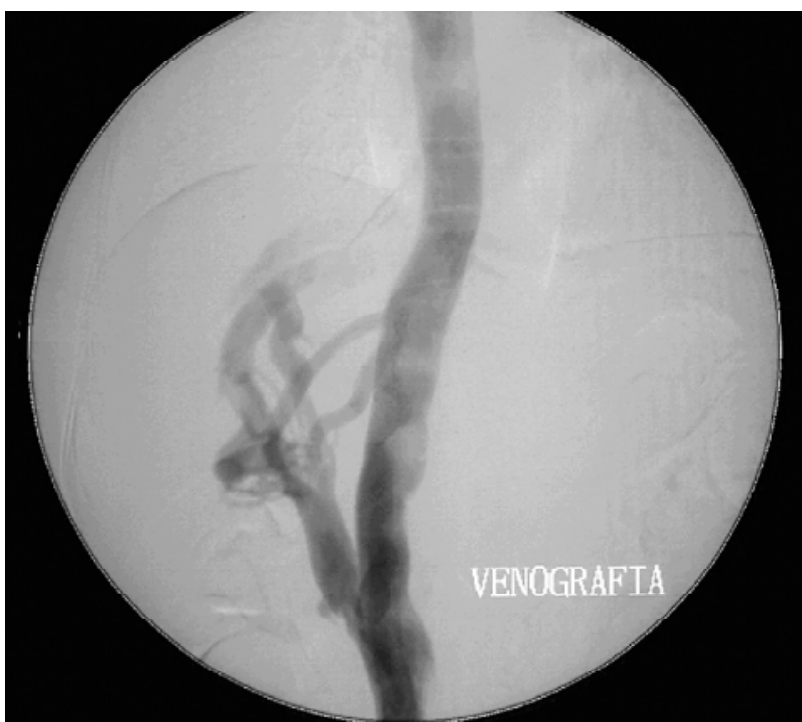

Figura 2 - Ausência deveia cava inferior supra-renal eveia ázigo vicariante

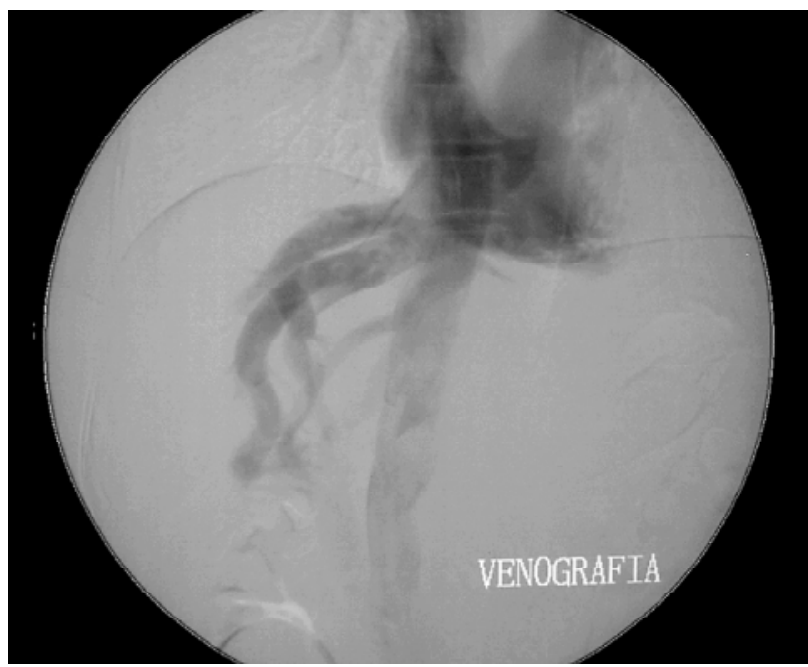

Figura 3 - V Veias supra-hepáticas drenando para 0 átrio direito, veiaázigo drenando paraacava superior

A agenesia da veiacavainferior érelatada como uma anomalia rara (menos de 1\% da população), resultante dedesenvolvimento embrionário aberrante ${ }^{1-7}$. H árelatos de que malformações da veia cava compreenderiam $5 \%$ de todas as TVP em pacientes com menos de 50 anose $16 \%$ das TVP deilíaca nesta mesma faixa etária 8 . A etiologia da TVP em pacientes jovens geralmente é uma anormalidade da coagulação congênita ou adquirida, doença imunológica ou neoplasia, mas, com as novas técnicas deimagem, asmalformações da veiacava são um novo fator a ser considerado ${ }^{8}$.
0 defeito embrionário, nesses casos, éresultante de uma atrofia inadequada das veias primitivas (pós-cardinais, subcardinais e supracardinais) - no caso da veia cava inferior supra-renal, um defeito da veia subcardinal direita de seanastomosar com os sinusóides hepáti$\cos ^{1,2,4,6,7,9,10}$. A drenagem venosa da parteinferior do corpo retorna ao coração pelo sistema ázigo vicariante até a veia cava superior e pelas veias hepáticas que drenam diretamenteparao átrio direito ${ }^{4,6,9}$. A veia cava inferior infra-renal pode ser direita, esquerda ou dupla (Figura 4$)^{6}$. Pode estar associada a outras anormalidades cardíacas ou abdominais, mas os pacientes que não possuem essas outras anormalidades são tipicamente assintomáticos ${ }^{1,2,6,9,10}$.

A agenesia de veia cava é considerada fator de risco para TVP por facilitar a estase venosa e aumentar a pressão no sistema venoso dos membros inferiores. Poucoscasosestão descritosnaliteraturamédica ${ }^{1,3,8,10}$.

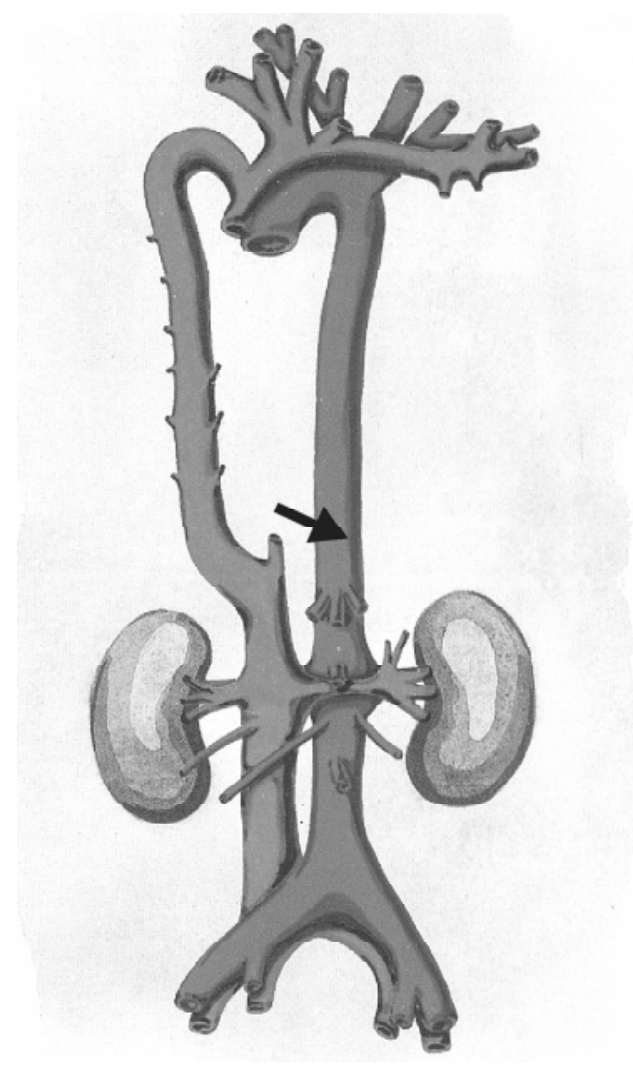

Figura 4 - Esquema gráfico da interrupção da veia cava inferior supra-renal e sua continuidade pela veia ázigo (adaptado de Latorre ${ }^{6}$ ) 
A maior parte dos casos descritos foi diagnosticada em pacientescom menosde 30 anosecom trombosedaveia ilíaca ${ }^{8}$.

Com relação ao tratamento, raramente é recomendada a cirurgia ${ }^{1}$. Como a paciente não apresentou novos episódios de TVP após a anticoagulação, e a recidiva da TVP em pacientes sem anticoagulação e malformações da veia cava éal ta ${ }^{8}$, foi decidido reiniciála. A paciente foi anticoagulada com varfarina, $5 \mathrm{mg}$ ao dia, e recebeu alta hospitalar. D everá, em princípio, permanecer anticoagulada. Além disso, recebeu orientações para evitar outros fatores de risco para TVP, como o uso dehormônios ou imobilização prolongada.

Concluímos que, nos casos envolvendo pacientes jovens, sem fatores de risco para TVP e onde os exames clássicos para investigação de trombofilias estão inalterados, devem ser excluídas malformações do sistema venoso cava como causa de TVP de repetição dos membros inferiores.

\section{Referências}

1. Cho BC, Choi HJ, Kang SM, et al. Congenital absence of inferior vena cava as a rare cause of pulmonary thromboembolism. Yonsei M ed J. 2004;45(5):947-51.

2. Fortaleza SCB, C ortez AP, Furtado LET A, et al. Agenesia de veia cava inferior na síndrome unha-patela. J Vasc Bras. 2004;3(3):273-6.

3. L ane D A. C ongenital hypoplasia of the inferior vena cava: an underappreciated cause of deep venous thromboses among young adults. M il M ed. 2005;170(9):739-42.
4. Pearce W H, Yao JST, Baxter BT, M CC arthy WJ. Venous angiodysplasia and venous malformation. In: Bergan JJ, Yao JST . V enous disorders. Philadel phia: W B Saunders; 1991. p. 362.

5. Valentine RJ, Wind GG. Anatomy of commonly exposed arteries. In: Rutherford RB. V ascular surgery. Philadelphia: W B Saunders; 2000. p. 648-660. Disponível em: http:// www.sbacvsp.org.br/medicos/biblioteca/indiceslivros/ rutherford.htm.

6. Latorre J. M alformaciones congénitas. In: Latorre Vilallonga JL. Sector Iliocava. Anatomía, fisiopatología, exploraciones y tratamiento. Barcelona: Edika-M ed; 1993. p. 75-94.

7. Cotta-PereiraG . Embriologiaehistologiado sistemavascular. In: Brito CJ. Cirurgia vascular. Rio de Janeiro: Revinter; 2002. p. 17-20.

8. G arcia-Fuster M J, Forner M J, Flor-LorenteB, Soler J, Campos S. Inferior vena cava malformations and deep venous thrombosis. Rev Esp Cardiol. 2006;59(2):171-5.

9. Kempinas W G. 0 desenvolvimento do sistema vascular. In: M affei FH A, Lastória S, Yoshida WB, Rollo HA. D oenças vasculares periféricas. 3rd ed. São Paulo: M edsi; 2002. p. 16-7.

10. Castro FJ, Pérez C, N arváez FJ, et al. Agenesia de vena cava inferior como factor deriesgo detromboembolismo pulmonar. An M ed Interna (M adrid). 2003;20(6):304-6.

Correspondência:

T elmo P. Bonamigo

Rua C el. Bordini, 675/303

CEP 90440-001 - Porto Alegre, RS

Tel./Fax: (51) 3333.1642

E-mail: telmobonamigo@terra.com.br 